\title{
Nanomorphologies in Conjugated Polymer Solutions and Films for Application in Optoelectronics, Resolved by Multiscale Computation
}

\author{
Cheng K. Lee ${ }^{1}$ and Chi C. Hua ${ }^{2}$ \\ ${ }^{1}$ Research Center for Applied Sciences, Academia Sinica, \\ ${ }^{2}$ Department of Chemical Engineering, National Chung Cheng University,
}

Taiwan

\section{Introduction}

Conducting conjugated polymers, which provide flexibility as polymers as well as conductivity as metals, have nowadays become an essential solution-processable material for fabricating polymer light-emitting diodes (PLEDs) and plastic solar cells. In addition to the possibility of producing large-area thin films at room temperature, an appealing feature of exploiting long-chain organic semiconductors lies in the capability to fine-tune the optoelectronic behavior of solution-cast films by exploiting a broad variety of solvents or hybrid solvents in preparing the precursor solutions, later fabricated into dry thin films via spin coating or ink-jet printing. To improve the solubility in usual organic solvents, the polymers are often modified by grafting flexible alkyl or alkoxy side chains to the phenyl backbone, rendering the polymer chemical amphiphilicity. The semiflexible backbone and chemical amphiphilicity, in turn, give rise to a vast swath of single-chain and aggregation morphologies as different types of solvents are used to cast the polymer thin films, through mechanisms-generally referred to as the memory effect (Nguyen et al. 1999)-as schematically illustrated in Figure 1. Clearly, understanding how the above-mentioned material properties evolve during a practical processing is of paramount importance, yet this central goal remains challenging to conventional experimental protocols. Computation simulations, therefore, provide an important alternative by which in-depth information may be readily extracted that complement our knowledge from experimental characterizations, and thereby facilitates the pursuit of gaining practical controls over the molecular states of solution-cast thin films.

This monograph aims to provide a comprehensive review of recently developed multiscale computation schemes that have been dedicated to resolving fundamental material properties in conjugated polymer solutions and films; prospects on emerging opportunities as well as challenges for upcoming applications in the area of organic optoelectronics are also remarked. Utilizing a standard, widely studied, conjugated polymer - poly(2-methoxy5-(2'-ethylhexyloxy)-1,4-phenylenevinylene) (MEH-PPV; see sketches in Figure 3)-as a representative example, we introduce the fundamentals and protocols of constructing self- 
consistent, parameter-free, coarse-grained (CG) polymer models and simulation schemes capable of capturing single-chain and aggregation properties at various length/time scales pertinent to a wide range of experimental measurements, as depicted in Figure 2. Meanwhile, predictions on specific material properties are discussed in view of the central implications for understanding known, yet-unresolved, experimental features, as well as for unveiling molecular properties for innovatory purposes. The main text is so organized: Sec. 2 describes the details of four different molecular dynamics schemes that virtually constitute a versatile multiscale computation "network," which can be utilized in an economic way to gain practical access to fundamental single-chain and aggregation properties from solution to the quenching state for, in principle, any specific conjugated polymers and solvent systems. The major computational results are summarized and discussed in Sec. 3. Finally, Sec. 4 concludes this review by outlining some future perspectives and challenges that become evident based on the current achievements.

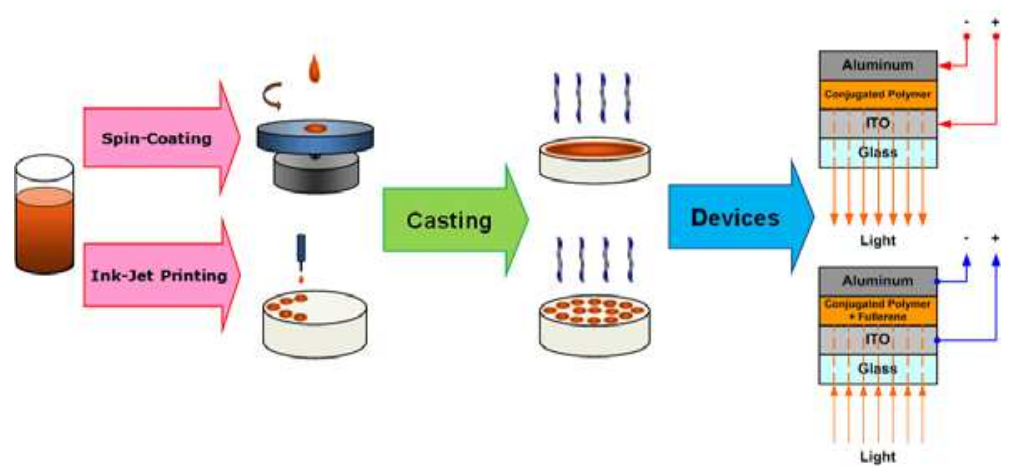

Fig. 1. Typical procedures for fabricating PLED devices or polymer-based solar cells.

\section{Simulation protocols}

Contemporary multiscale computations that concern polymer species typically begin with full-atom or united-atom molecular dynamics schemes-both are referred to as AMD scheme for simplicity - with incorporated interatomic force fields often built in a semiempirical manner for atoms or molecular units that share similar chemical structures and environments. Of course, these default force fields and associated parameter values should always be selected carefully and, if necessary, checked against the results of first-principles computation. The basic principle of constructing a CG polymer model is, once the polymer has been redefined by lumping certain molecular groups into single CG particles, self-consi-stent force fields that govern these CG particles may be built using AMD simulation data on the original, atomistic polymer model. For the case of intramolecular (bonded) CG potentials, the statistical trajectories of the redefined bond lengths and angles are first collected from the AMD simulation, and then Boltzmann inversions of their distribution functions are performed to evaluate the new potential functions which, in turn, are utilized in the corresponding CG simulation and the results checked against the AMD predictions for self-consistency; if necessary, repeat the above procedure until the imposed tolerance criteria are met. The situation is similar in constructing the intermolecular (non-bonded) CG potentials, except that one utilizes the so-called radial distribution functions (RDFs) and that 
a greater number of iterations are usually required because of a more pronounced effect of many-body interactions. Some of the details are provided in the following text, and abundant literature addressing these issues may be consulted (Carbone et al. 2010; Faller 2004; Müller-Plathe 2002; Noid et al. 2008; Padding \& Briels 2011; Tschöp et al. 1998).

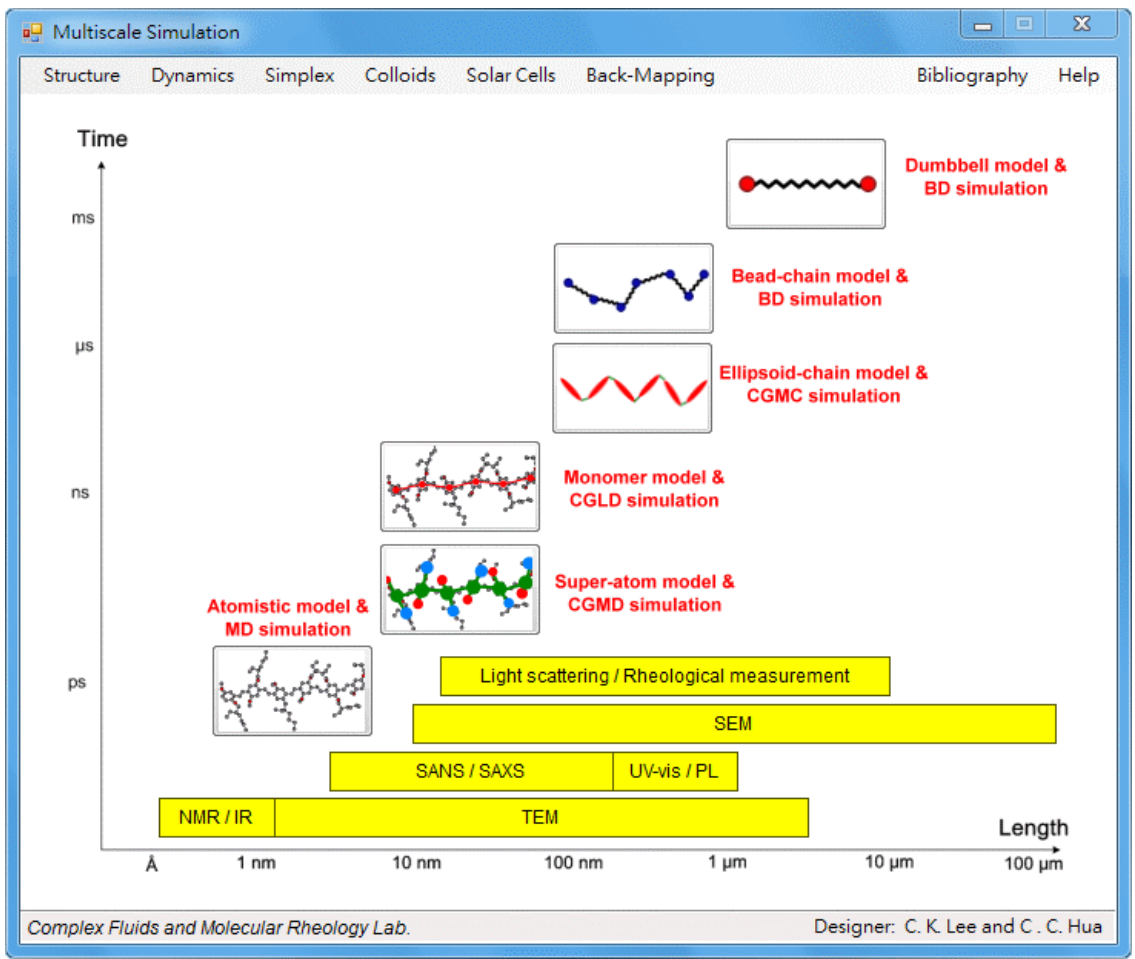

Fig. 2. Multiscale simulation schemes that provide molecular information at various length/time scales pertinent to a wide range of experimental measurements.

\subsection{Coarse-Grained Molecular Dynamics (CGMD) simulation}

The most primitive CG scheme for simulating a polymer solution is to explicitly retain the solvent molecules and treat them as usual CG particles as for the polymer molecule. In this way, the simulation of the CG system may be carried out by the same software package as for previous AMD simulations, provided the newly constructed bonded and non-bonded potentials for all CG particles. Figure 3 depicts how a MEH-PPV chain may be coarsegrained by introducing suitable "super-atoms" to represent essential molecular units -in this case, the repeating phenyl backbone unit and two asymmetric alkoxy side-chain groups. Likewise, solvent molecules are cast into single CG "beads" of similar size. All CG particles are mapped at the mass centers and converse the full masses of the molecular units they represent. As has been noted earlier, the next step involves rebuilding self-consistent, parameter-free, intramolecular potentials governing the CG particles by using the Boltzmann inversions of essential statistical trajectories gathered from AMD simulations of the original, full-atom or united-atom, representation of the model system: 


$$
U(z)=-k_{\mathrm{B}} T \ln P(z),
$$

where $k_{\mathrm{B}} T$ is the Boltzmann constant times the absolute temperature, and $P(z)$ is the probability distribution function of the independent variable $z$ (i.e., bond lengths or angles) redefined in the CG polymer model. Similarly, the RDFs retrieved from specially designed AMD simulations are adopted in the construction of intermolecular CG potentials. Subsequent iterations to ensure self-consistencies between AMD and CGMD simulations may be enforced by simplex optimizations:

$$
\begin{gathered}
f=\int_{0}^{\text {cutoff }}\left(U^{\mathrm{AMD}}(z)-U_{i}^{\mathrm{CGMD}}\left(z,\left\{p_{n}\right\}\right)\right)^{2} d z \rightarrow \min , \\
f=\int_{0}^{\text {cutoff }}\left(R D F^{\mathrm{AMD}}(r)-R D F_{i}^{\mathrm{CGMD}}\left(r, U_{i}^{\mathrm{CGMD}}\left\{p_{n}\right\}\right)\right)^{2} \mathrm{~d} r \rightarrow \min .
\end{gathered}
$$

If the usual 12-6 Lennard-Jones (LJ) type of intermolecular potentials are assumed for the CG particles, as in the present case, the initial guess may be obtained via the following relation: $\quad U_{i=1}^{\mathrm{CGMD}}(r)=4 \varepsilon\left[(\sigma / r)^{12}-(\sigma / r)^{6}\right] \approx-k_{\mathrm{B}} T \ln \left(R D F^{\mathrm{AMD}}(r)\right)$. The full set of parameters $\left\{p_{n}\right\}$ in this case denote the well depth $\varepsilon$ and the van der Waals diameter $\sigma$, and $i$ stands for the number of iterations attempted. An important advantage of the above choice is, in fact, that a simple mixing rule may be adopted to describe the pair potentials for unlike CG particles, thus saving a lot of computational effort. Justifications of such simplified treatment for the simulation systems under investigation have been discussed in earlier work (Lee et al. 2009; Lee et al. 2011).

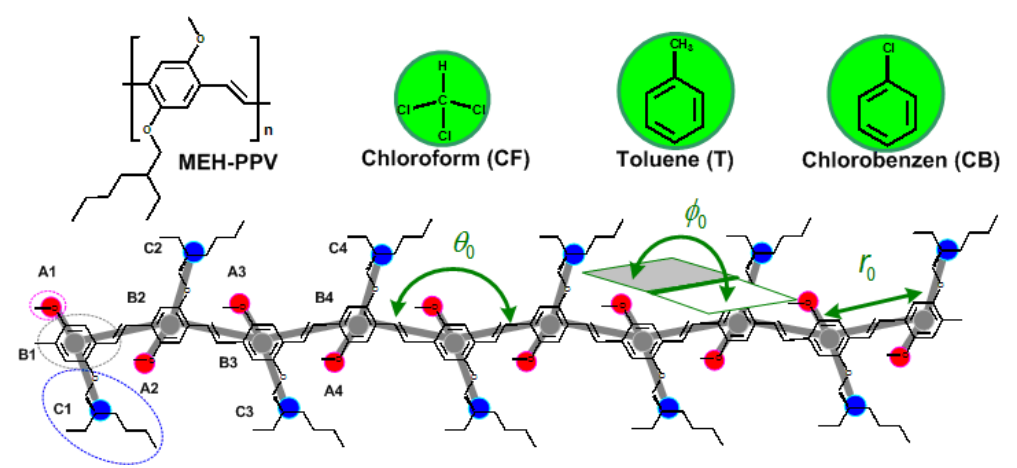

Fig. 3. Specifications of representative bond lengths and angles for the super-atom model of MEH-PPV, where B, A and C denote the aromatic backbone, short- and long-alkoxy side chains, respectively.

For the polymer model depicted in Figure 3, which represents the "finest" CG polymer model in this review article, the two side-chain groups are treated as independent CG particles so as to discriminate the chemical affinities of various types of solvent molecules with respect to different parts of the polymer chain. Moreover, tetrahedral defects (which represent a localized breakage of single/double-bond conjugation) are incorporated and 
assigned uniformly to every 10 repeating units on the polymer backbone, in order to realistically capture the collapsed morphologies of real synthesized chains during the quenching process. Simulation results based on this CG solution system have been obtained for a 300-mer MEH-PPV, close to the chain length of a commercial sample commonly used in experiment. Both AMD and CGMD simulations utilized the $N P T$ ensemble at $T=298 \mathrm{~K}$ and $P=1 \mathrm{~atm}$, with the same software package (Forester \& Smith 2006) where the incorporated force fields (Mayo et al. 1990) were noted to lead to generally good agreement with known experimental features of MEH-PPV solution (Lee et al. 2008), as well as with force fields (particularly for torsional angles) suggested by first-principles computations (De Leener et al. 2009).

\subsection{Coarse-Grained Langevin Dynamics (CGLD) simulation}

As our primary interest turns to large-scale material properties, such as the morphologies of long single chains or interchain aggregates, the CGMD scheme described above becomes inefficient because most of the computational times must be devoted to the uninterested, generally overwhelming in number, solvent molecules. A classical solution to this problem is treating the solvent as a continuum thermal bath and, accordingly, modifying the Newton's equations of motion to be the Langevin ones - the solution schemes of which are often referred to as Brownian dynamics-by adding self-consistent frictional drag and thermal Brownian forces. Conventional Brownian dynamics simulations, however, differ distinctively from the one introduced below in both the degree of coarse-graining and the retrieval of parameter values for drag coefficient. More specifically, the drag coefficient used in conventional Brownian dynamics is typically derived from the Einstein-Stokes relation for large, Brownian particles, and usually bears no direct link with the molecular attributes of the specific polymer-solvent pair under investigation. In fact, at the previous level of coarse-graining, the frictional drags have been treated as dissipative forces, independent of the solvent quality which might be accounted by the "excess" non-bonded bead interactions. Recently, considering a CG polymer model of MEH-PPV as depicted in Figure 4, we have proposed strategies that help reconcile the dilemma noted above for usual Brownian dynamics schemes for dilute solution (Lee et al. 2008). The central idea is that, instead of assuming the Einstein-Stokes relation - which strictly applies only to Brownian particles that are sufficiently larger than the solvent molecules-the diffusivity of a CG particle representing a monomer unit, $D$, was "measured" directly from an AMD simulation, and the frictional drag coefficient, $\varsigma$, was later evaluated from the more fundamental Einstein equation, $\varsigma=k_{\mathrm{B}} T / D$. As usual, this allows the Brownian forces to be constructed selfconsistently from fluctuation-dissipation theorem. The resulting Langevin equation bears the form

$$
m_{i} \frac{d^{2} \mathbf{r}_{i}}{d t^{2}}=-\varsigma_{i} \frac{d \mathbf{r}_{i}}{d t}+\sum_{j} \mathbf{F}_{i j}+\xi_{i},
$$

where $m_{i}$ and $\mathbf{r}_{i}$ denote the mass and positional vector of the $i$ th bead on a certain polymer chain, respectively, $\sum_{j} \mathbf{F}_{i j}$ and $\xi_{i}$ represent the sum of the conservative forces (i.e., the intra- and intermolecular forces) and the random force, respectively, acting on the same bead, and $\varsigma_{i}$ is the frictional drag coefficient. The following expression of Brownian forces 
with a Gaussian statistics can be constructed: $\left\langle\xi_{i}\right\rangle=\mathbf{0}$ and $\left\langle\xi_{i}(t) \xi_{j}(t)\right\rangle=2 \varsigma_{i} k_{\mathrm{B}} T \delta_{i j} \mathrm{I}$, where the broken brackets denote taking the ensemble average of the quantity within them, $\mathbf{I}$ is a unit tensor, and $\delta_{i j}$ is the Kronecker delta function.

Significantly, the results shown in Table 1 suggest that the CGLD scheme so constructed is able to capture both the dynamic and structural properties of single MEH-PPV chains, and the computational efforts so saved are enormous. To gain a better feeling, we mention that for the results shown in Table 1, it takes ca. 36 hrs of the CGMD simulation with 4 CPUs running in parallel, while it requires only about 10 minutes for the CGLD simulation executed in a single-CPU personal computer. As an important consequence, longer MEHPPV chains (i.e., above 300-mers), their supramolecular aggregates, and longer real times (up to several hundred nanoseconds) may be simulated in a single-CPU personal computer.

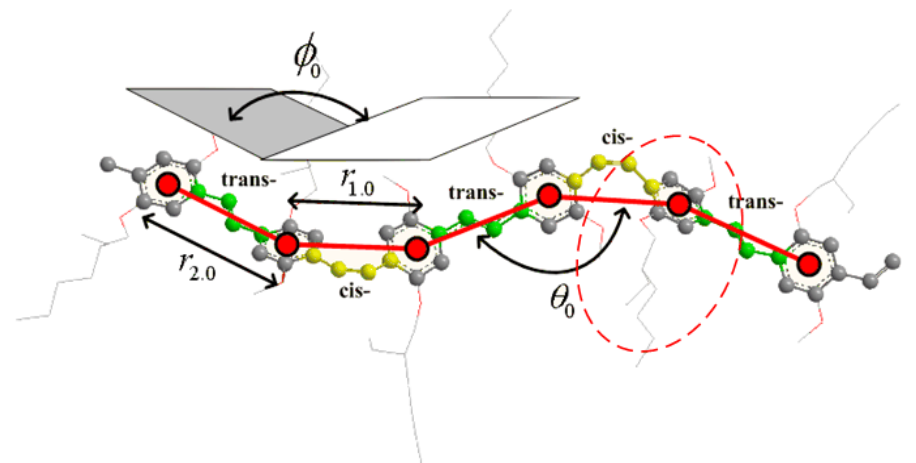

Fig. 4. Specifications of a few representative bond lengths and angles for the monomer model of MEH-PPV (circles).

\begin{tabular}{|cll|}
\hline 100-mer MEH-PPV & Radius gyration $(\AA)$ & Diffusivity $\left(\mathrm{m}^{2} / \mathrm{s}\right)$ \\
\hline CGMD & $R_{\mathrm{g}, \mathrm{MT}}=26.77 \pm 1.42$ & $D_{\mathrm{MT}}=2.97 \times 10^{-10}$ \\
\hline CGLD & $R_{\mathrm{g}, \mathrm{MT}}=26.48 \pm 1.02$ & $D_{\mathrm{MT}}=2.62 \times 10^{-10}$ \\
\hline CGMD & $R_{\mathrm{g}, \mathrm{MC}}=33.40 \pm 1.19$ & $D_{\mathrm{MC}}=5.90 \times 10^{-10}$ \\
\hline CGLD & $R_{\mathrm{g}, \mathrm{MC}}=34.03 \pm 0.97$ & $D_{\mathrm{MC}}=4.66 \times 10^{-10}$ \\
\hline
\end{tabular}

Table 1. Comparisons between CGLD and CGMD simulations for the predicted radius of gyration and center-of-mass diffusivity in MEH-PPV/toluene (MT) or MEH$\mathrm{PPV} /$ chloroform (MC) solution.

\subsection{Coarse-Grained Monte Carlo (CGMC) simulation}

Considering the planar or ellipsoidal backbone segments of typical conjugated polymers, the classical Gay-Berne (GB) potential (Gay \& Berne 1981) seems ideal for describing the segmental interactions of large oligomer units. The GB potential and the associated ellipsoidchain model, as sketched in Figure 5, is appealing also in that synthesized defects, tetrahedral 
ones in particular, may be naturally embodied in the form of connecting springs between any two adjacent ellipsoid segments. If ten percent of such defects were assumed, for example, each ellipsoid effectively represents a 10-mer MEH-PPV segment, thus greatly enlarging the degree of coarse-graining. Given that the GB potential is able to treat the effects of molecular anisotropy in both attractive and repulsive interactions in an explicit and computationally efficient manner, it has nowadays become a standard model for studying the phase behavior and microstructures of liquid crystals, anisotropic colloids and liquid crystalline polymers, albeit most of the early applications were restricted to small molecules with aspect ratios generally below five. As addressed in an early work (Lee et al. 2010), applying the GB potential for a semiflexible, large oligomer species like a 10-mer MEH-PPV requires special cares in establishing the potential of mean forces (PMFs) between two ellipsoids, as well as in fixing simultaneously a large set of floating parameters. The functional form, the principal set of parameters and their determinations can be found elsewhere (Lee et al. 2010).

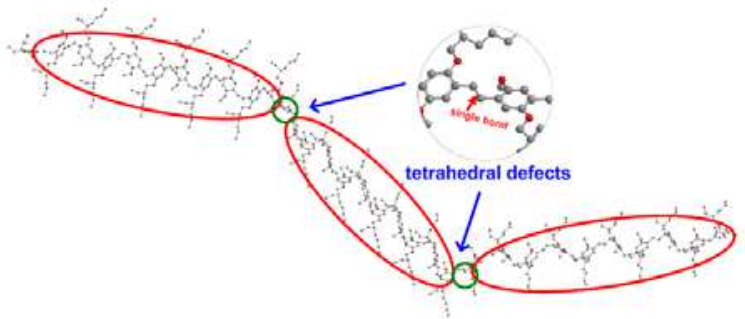

Fig. 5. Atomistic model representation and the ellipsoid-chain model (line contour) for a MEH-PPV oligomer with uniformly distributed tetrahedral defects.

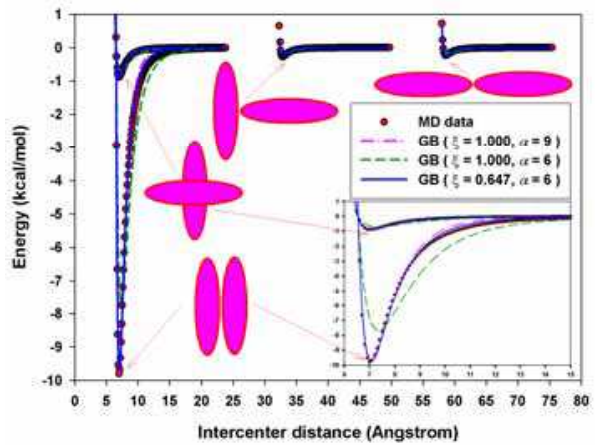

(a)

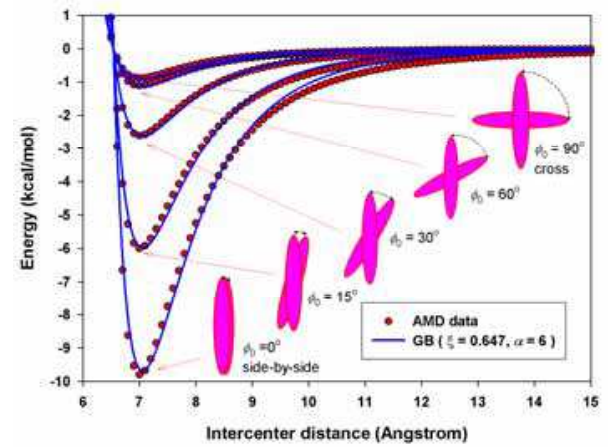

(b)

Fig. 6. Comparison of the predicted potential curves between AMD computations (symbols) and the parameterized GB model (lines) for (a) four representative arrangements and (b) various other arrangements of two like MEH-PPV oligomers.

Figure 6(a) shows how the parameter values in the GB potential may be determined based on the PMFs found in the AMD simulations for four representative mutual alignments of two like ellipsoids; the significances of the symbols appearing in the inset and the comparisons made can be found in early discussion (Lee et al. 2010). Figure 6(b) confirms 
that the GB potential so parameterized can also capture quite well the PMFs of other mutual alignments that are deemed the most important to capture for a dense or condensed system. Unlike the CG polymer models described earlier, however, the segmental interactions described by the GB potential cannot be easily cast into usual equations of motion, and hence, only results from Monte Carlo simulations have been reported. Moreover, since the potential functions were previously parameterized for vacuum environment, the effects of solvent quality must be further accounted for. For the latter aspect, we have recently devises a similar procedure in building the PMFs for a pair of ellipsoids suspended in specific solvent media, and the ellipsoid-chain model so constructed leads to good agreement for the predicted solvent qualities as compared with the CGLD simulation results; more details will be published in a future work.

\subsection{Brownian dynamics (BD) simulations of bead-spring chain and dumbbell models 2.4.1 Bead-spring chain models}

As mentioned above, a notable drawback of the GB potential and the associated ellipsoidchain model is that it is inherently more compatible with MC schemes and, hence, is not convenient for investigating dynamic properties. In addition, polymer segments with an aspect ratio as high as 10, for example, can easily be trapped in local minima in dense or condensed systems in a MC simulation. An alterative way to attain a similar level of coarsegraining, while compatible with usual dynamics schemes, is to resort to conventional beadspring models, such as freely rotating (FR) chain and freely joined (FJ) chain. These kinetic models have a long history of being deployed to investigate a wide range of polymeric and biological systems. Figure 7 shows how a MEH-PPV chain may be coarse-grained into consecutive bead-spring segments, each essentially modeling the end-to-end orientation and separation of a certain group of monomer units. Depending on the number of monomers included in such a segment with respect to that constituting a Kuhn segment, a FR chain or FJ chain can be selected as the CG polymer model, and the implementation of Brownian dynamics schemes is straightforward. For instance, if the simulation aims to capture the local rodlike structure as well as the global coil-like feature of a sufficiently long MEH-PPV chain, the FR chain model may be adopted for this purpose. On the other hand, the FJ chain model will be more efficient as the morphologies of large aggregate clusters are of major concern. In practice, both models can be utilized interchangeably in the forward/backward mappings to compromise efficiency and efficacy, as we discuss later.

A serious problem arises, however, while constructing non-bonded bead potentials, and this foreseen difficulty is reminiscent of the inherent inadequateness of mapping ellipsoidal or rodlike segments of a semiflexible chain onto spherical beads. Thus, with increasing degree of coarse-graining, determinations of the effective bead diameter inevitably become ambiguous. This situation clearly reflects the tradeoff as one picks the bead-spring models as an expedience in lieu of the much more complicated, yet realistic, ellipsoid-chain models for semiflexible chains. In a recent work, we proposed strategies that utilize material properties of intermediate length scales - e.g., the Kuhn length and polymer coil density - that can readily be known from finer-grained simulations, along with a single set of small angle neutron scattering (SANS) data, to parameterize the bonded and non-bonded potentials of a FR chain, with the latter assuming a LJ form (Shie et al. 2010). In the next section, we examine the performance of Brownian dynamics simulations based on the FR chain model in describing large-scale aggregation properties, which also manifest themselves in the same set of SANS data previously used to determine the parameters for single chains. 


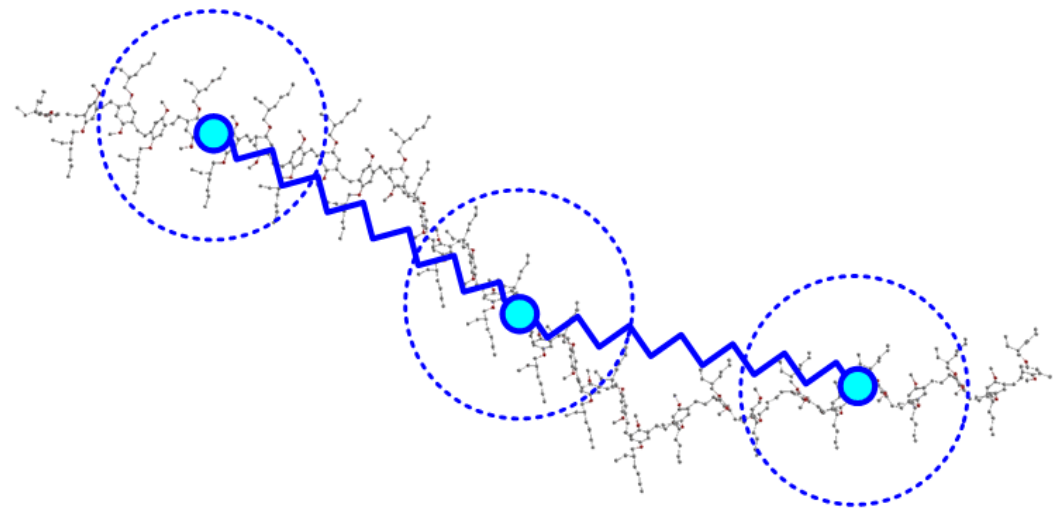

Fig. 7. Atomistic model versus bead-chain model representation for a MEH-PPV oligomer.

\subsubsection{The dumbbell model}

The dumbbell model, as depicted in Figure 8, in conventional polymer kinetic theories has been to mimic the end-to-end orientation and separation of a polymer chain without account of any intermediate details as may be captured by a bead-spring chain model introduced above. In both cases of the FJ and dumbbell models, there exist standard classical theories relating the spring potential with the number of Kuhn segments belonging to a chain segment or the dumbbell. In simulating an aggregated polymer system, the nonbonded bead potential plays an important role, too, and must be constructed on a sound basis. The protocols proposed in an early work (Shie et al. 2007) resemble the one described in Sec. 2.3 for two ellipsoid segments, except that the PMFs of two previously parameterized FJ chains were used for this purpose. The dumbbell model so constructed is especially useful for simulating large-scale aggregation properties, as the detailed chain conformation is of less concern, or when the simulation results are meant to be back-mapped to the FJ or FR models to expedite the subsequent equilibration.

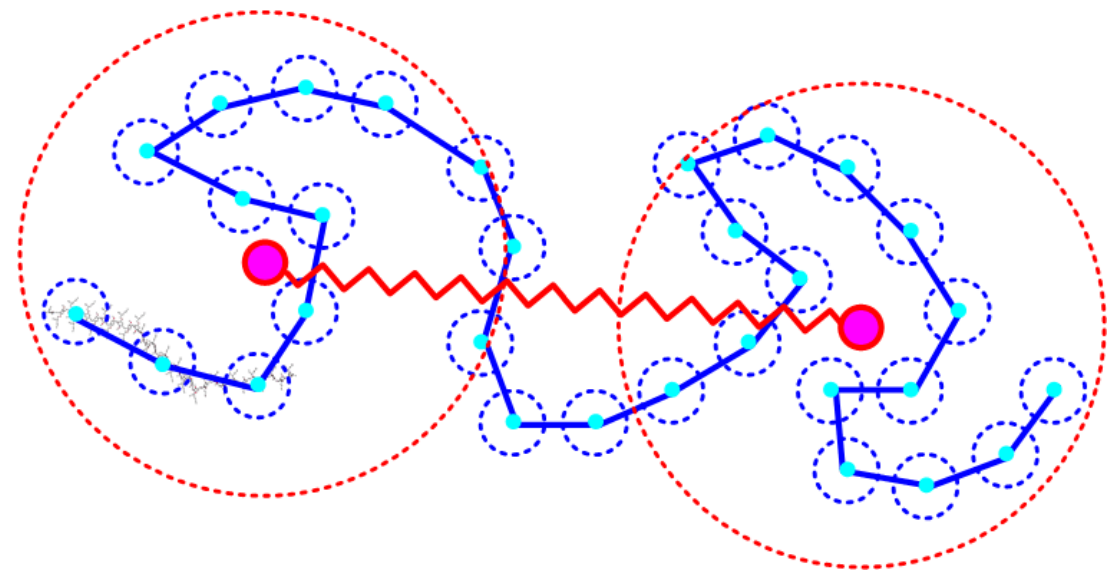

Fig. 8. Bead-chain and dumbbell model representations for a single MEH-PPV chain. 
Before closing this introductory section for various CG polymer models and simulation schemes for MEH-PPV, it is very important to keep in mind that their conventional counterparts have mostly been used for more qualitative purposes, often without specifications of the particular polymer-solvent considered. In contrast, the cases of our current interest are meant to be predictive in the first place. It also appears that no early studies have covered such a wide range of CG models and simulation schemes for a single polymer system as have been demonstrated for MEH-PPV. The last perspective, intriguingly, points to the open opportunity of utilizing multiscale schemes to capture real large-scale material properties of specific macromolecular systems.

\section{Results and discussion}

In this section, the simulation results are presented according to increasing degree of coarsegraining, similar to the way the models have been introduced above. It can be seen that large-scale material properties become progressively accessible as some molecular details become irrelevant and, hence, more coarse-grained views may be adopted. To delve into how material properties evolve during a practical processing, it is also instructive to understand first elementary solution properties before their impacts on the eventual quenching state are scrutinized.

\subsection{Single-chain properties in binary solvent media}

The polymer model depicted in Figure 3 and the corresponding CGMD simulation are especially relevant to resolving the detailed polymer-solvent interactions in various solvent or hybrid-solvent systems; more details can be found in an early work (Lee et al., 2011). In the literature, some confusion prevailed regarding the "solvent quality" for an amphiphilic conjugated polymer like MEH-PPV, and further clarification on this fundamental issue for polymer solution would be possible if a better understanding into such interactions can be gained in an unequivocal way. For instance, an aromatic solvent, such as toluene, is expected to be attractive to the phenyl backbone of MEH-PPV and, intuitively, might be conjectured to serve as a better solvent than an aliphatic solvent, such as chloroform, which may be attractive to the alkoxy side chains at best. The interesting question is: which of the two helps bolster a relatively expanded chain conformation or, in a statistical sense, larger mean coil size? The situation is even complicated, more intriguing as well, as two distinct solvents are concurrently present in a hybrid-solvent system. In both cases, it should be evident that treating the side-chain and backbone molecules as different CG particles are essential to address theses issues.

Figure 9 shows the predicted mean radii of gyration as functions of the solvent composition for two representative sets of binary solvents, chloroform (CF)/toluene (T) and chloroform/chlorobenzene (CB). As far as single-solvent systems are concerned, the apparent solvent quality clearly follows the ordering $\mathrm{CF}>\mathrm{CB}>\mathrm{T}$. Interestingly, the previous prediction is at odd with the naïve, prevailing notion that aromatic solvents which bear better chemical affinity with the backbone molecules - would result in a better solvent quality. As we turn our attention to the more complicated, binary-solvent systems, Figure 9 reveals another significant feature that cannot be foreseen on the basis of the results just revealed for single-solvent systems. It can be seen that the mean coil size, and thus the apparent solvent quality, is not a monotonic function of the solvent composition, and there appears to be an optimum mixing ratio in each case, i.e., $\mathrm{CF} / \mathrm{T}=1: 1$ or $\mathrm{CF} / \mathrm{CB}=2: 1$ in number density, that renders the best solvent quality. Some representative snapshots given in Figure 10 are instructive in light of what the overall chain conformation might look like in each of 
these systems. It is of interest to note the highly extended chain conformations corresponding to the two optimum solvent qualities. Noticing that the detailed chain conformation in dilute solution is dictated by localized polymer-solvent interactions, we next scrutinize the simulation results that offer the relevant details - features that cannot be assessed by experimental protocols.

Because the two hybrid-solvent systems exhibit essentially the same trends, the following discussion is focused on the CF/CB solvent system of MEH-PPV. Figure 11(a) plots the local ratio of the two solvent species, normalized by the bulk one, as a function of the normal distance away from the polymer backbone, as schematically illustrated in the same figure.

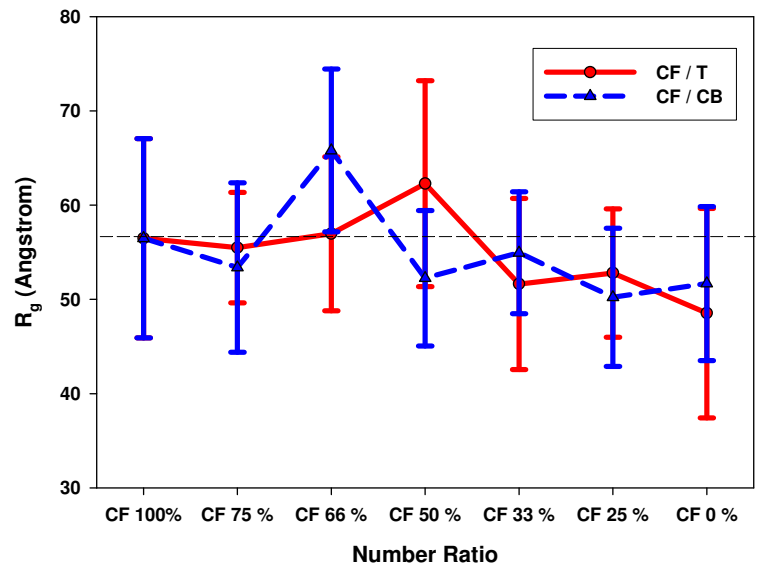

Fig. 9. The predicted mean radius of gyration for single 300-mer MEH-PPV chains in singleor binary-solvent system at $298 \mathrm{~K}$ and 1atm. The lines are used to guide the overall trend.
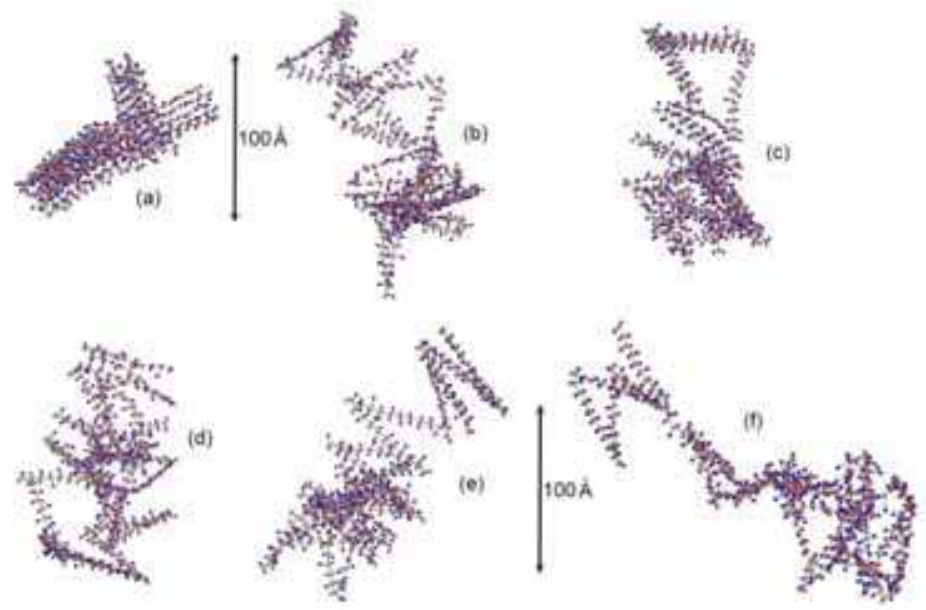

Fig. 10. Snapshots of MEH-PPV chain conformations in (a) quenching system, (b) chloroform, (c) toluene, (d) chlorobenzene, (e) chloroform/toluene=1:1 and (f) chloroform/chlorobenzene $=2: 1$. 
It can be seen that, within a thin shell of thickness ca. $3 \mathrm{~nm}$, local solvent compositions differ noticeably from the bulk one. In particular, at a mixing ratio of $C F / C B=2: 1$, which corresponds to an optimum solvent quality in Figure 9, the (positive) deviation seems to be the most prominent. The observation that local CF population could substantially outnumber that in the bulk phase is clearly suggestive of certain coupling effects that, in turn, are sensibly affected by the blending ratio of the two solvent species.

Figure 11(b) shows the corresponding RDFs, and the results for single-solvent CF system are also included for comparison. Several interesting features can be noted immediately. Firstly, the first (dominant) peaks in the RDFs clearly indicate that while $\mathrm{CF}$ molecules are considerably more attractive to the alkoxy side-chain units (C) of MEH-PPV, CB molecules are slightly more attractive to the backbone (B), as might be expected using chemical affinity arguments. Comparing the results with those for pure CF solvent system, however, reveals that $\mathrm{CB}$ molecules have a drastic impact on the peak height of the $\mathrm{C}-\mathrm{CF}$ pair distribution. That is, without the presence of $\mathrm{CB}$ molecules, $\mathrm{CF}$ is normally depleted from the polymer territory, possibly to avoid the backbone molecules. Further, an intriguing feature is that both curves of C-CF and B-CB display conspicuous oscillations beyond the first peak, a phenomenon rarely observed with single-solvent systems. The sketch given in the same figure suggests that this peculiar feature could be indicative of a somewhat ordered, latticelike or layered structure of solvent molecules encompassing the polymer units that are attractive to them.

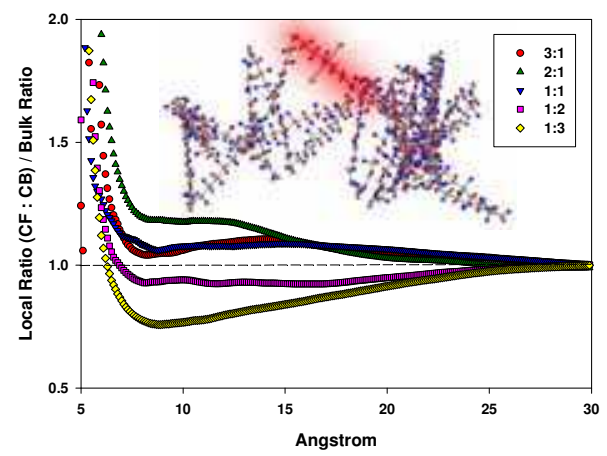

(a)

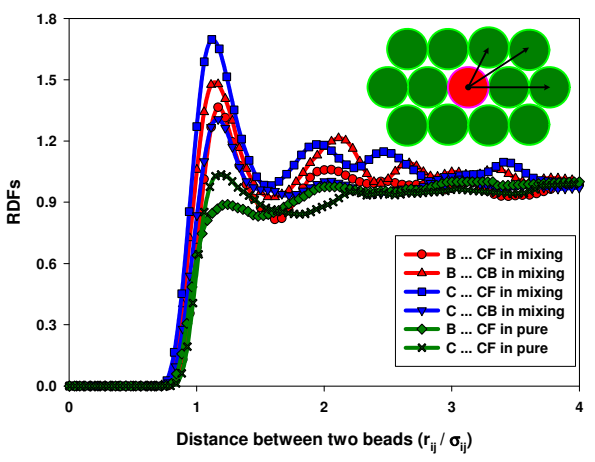

(b)

Fig. 11. (a) Solvent particle distributions measured at a normal distance away from the polymer backbone in the CF/CB binary-solvent medium of MEH-PPV. (b) The RDFs (for $\mathrm{CF} / \mathrm{CB}=2: 1$ ) reflecting the distributions of solvent molecules with respect to the backbone (B) or the long-alkoxy side chain (C) of the polymer; the results for single-solvent CF system are also shown for comparison. The distance in (b) has been normalized using the mean van der Waals diameters of the two CG particles involved.

We suggest the following interpretations of the phenomena noted above. The role played by CB molecules in the CF/CB hybrid solvent system of MEH-PPV is twofold: They attract, and thus "stabilize," the MEH-PPV backbone preventing too collapsed a chain conformation, especially with the presence of the "disliked" CF molecules that are to be avoided by the phenyl backbone. On the other hand, they help shield the "repulsions" directly between $\mathrm{CF}$ and MEH-PPV backbone, thus encouraging immigration of $\mathrm{CF}$ 
molecules into the shell regime to better attract the side-chain groups. Altogether, this sophisticatedly compromised, local molecular environment warrants the sustainability of exceptional, highly extended MEH-PPV chains-which obviously require a free exposure of both the side-chain and backbone units that can only be fulfilled in a hybrid-solvent medium for an amphiphilic polymer like MEH-PPV. Moreover, compared with toluene (T) molecules, the somewhat amphiphilic attribute of $\mathrm{CB}$ is expected to be a superior "mediator" to play the essential roles as suggested above, besides its better affinity to the CF molecule as well. Note, however, that as $\mathrm{CF}$ molecules progressively migrate into the boundary layer regime, they must do so against the bulk osmotic pressure, until an eventual balance between the two "phases" has been established. Thus, the peculiar features manifested by Figures 9-11 may be perceived as arising from a subtle balance between local-phase chemical affinities and bulk-phase osmotic pressure - the latter being basically entropic in nature.

\subsection{Structural and dynamic properties of single chains and interchain aggregates}

The central difference between the polymer models depicted in Figures 3 and 4, respectively, lies in that each repeating unit, including the bulky side-chain groups, is treated as a single CG particle in the latter representation, so that the corresponding CGMD or CGLD simulations become more efficient, especially for studying long single chains or interchain aggregates. Of course, the model becomes inadequate, though, for hybrid solvents as discussed above. As an example that demonstrates the robustness of CGLD simulations, Figure 12 shows the mean end-to-end distance of MEH-PPV for chain lengths up to 500 repeating units, which seems sufficient to determine the solvent quality exponents for the two solvents examined, i.e., chloroform and toluene.

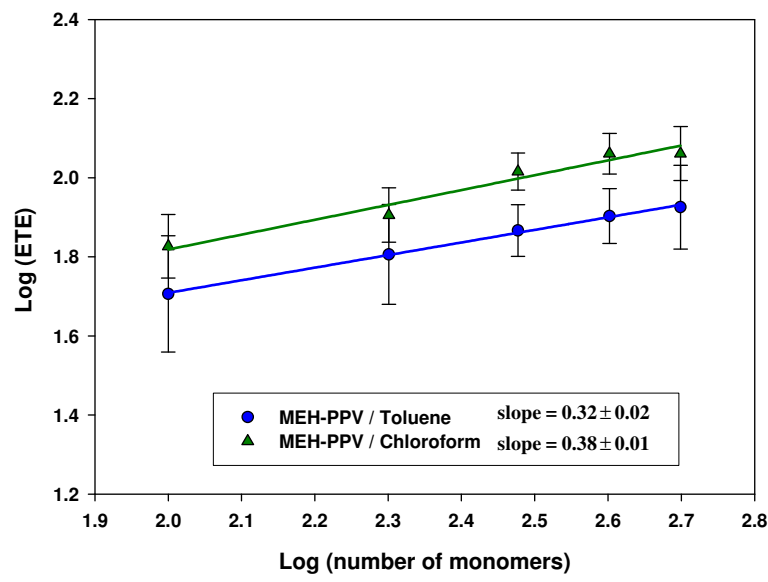

Fig. 12. The scaling law of the mean end-to-end distance (ETE) as functions of molecular weight (number of monomers per chain) for single MEH-PPV chain in two different solvent systems.

For long, the solvent quality of MEH-PPV solutions remained somewhat mysterious, due to the fact that experimental protocols, such as viscometric measurements and light scatterings, commonly purporting to assess this fundamental quantity for dilute solution become ineffective because of a great tendency for the polymer chains to associate and form interchain aggregates even at large dilution. Under this circumstance, computer simulations 
become indispensable to extract information for such analysis. The results shown in Figure 12 yield a solvent quality exponent of 0.38 for chloroform and 0.30 for toluene, suggesting that both are rather poor solvents for MEH-PPV. Given that chloroform is arguably the best solvent known so far for MEH-PPV, the interesting implication appears to be that no solvents can actually be "good" for this amphiphilic conjugated polymer, which requires a scaling exponent above 0.5 - the theta solvent condition. This central implication explains why intra- and interchain aggregates prevails even at large dilution.

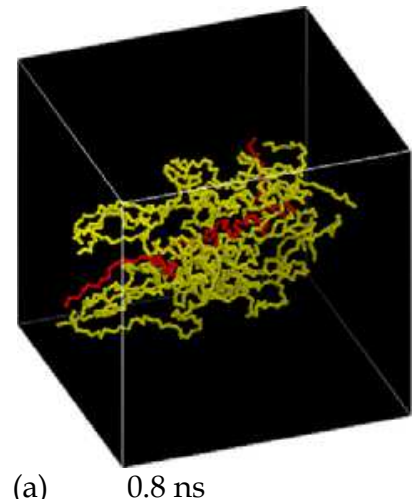

(a)

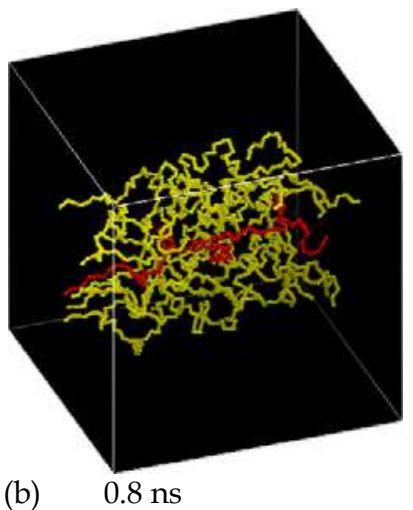

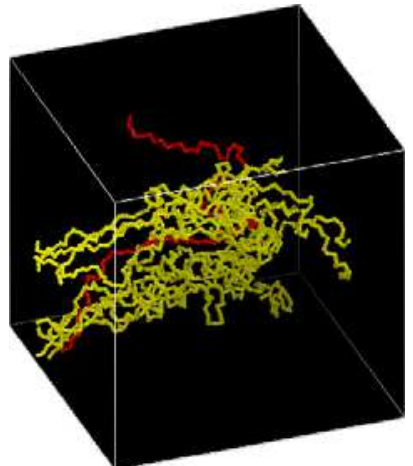

$2.4 \mathrm{~ns}$

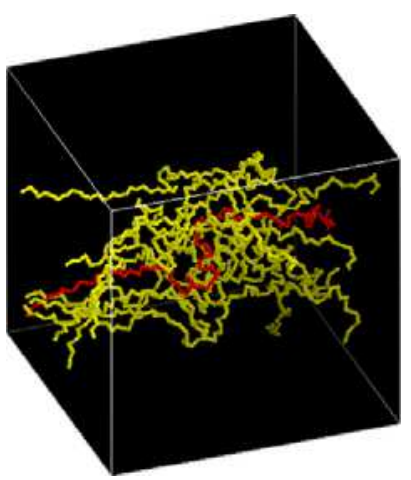

$2.4 \mathrm{~ns}$

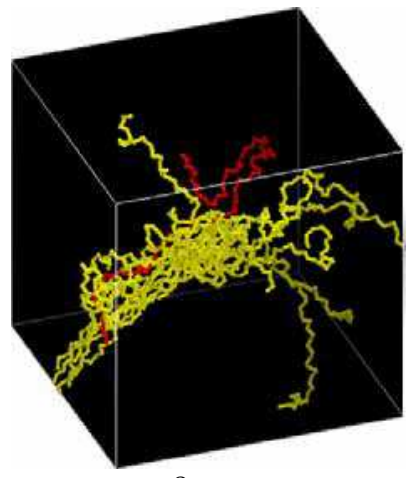

$8 \mathrm{~ns}$

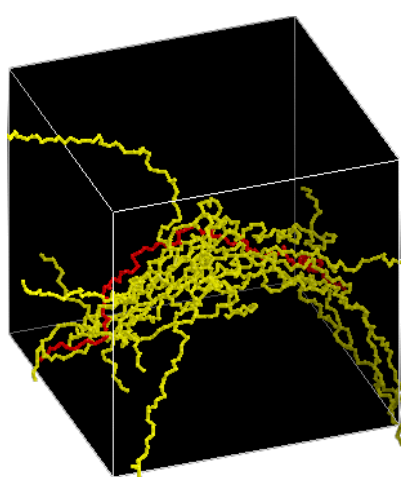

$8 \mathrm{~ns}$

Fig. 13. Snapshots of ten MEH-PPV chains collapsing into an aggregate cluster in (a) toluene or (b) chloroform at various times at $T=298 \mathrm{~K}$ and $1 \mathrm{~atm}$, where all chains were initially placed in parallel and assumed a fully extended chain configuration. The simulation time has been re-scaled to coincide with the real, AMD time.

Recently, SANS has been utilized to assess the Kuhn length of MEH-PPV in solution, by assuming that this localized chain property is unaffected by segmental or interchain aggregation (Ou-Yang et al. 2005). Thus, it is of interest to compare the simulation results with the experimental estimate. The persistence length of MEH-PPV is estimated in the CGLD simulation to be $65.1 \pm 11.8(\dot{\mathrm{A}})$ in toluene and $73.3 \pm 12.5(\dot{\mathrm{A}})$ in chloroform, which correspond to the mean end-to-end distance of about $25 \mathrm{MEH}-\mathrm{PPV}$ monomers, or 50 monomers in a Kuhn segment-if a ratio of two is assumed for these quantities. For 
comparison, we note that light scattering data have suggested a value of ca. $60 \AA$ in p-xylene (Gettinger et al. 1994), while SANS data yielded $87.5 \AA$ in chloroform (Ou-Yang et al. 2005). The agreement between simulation and experiments may be deemed fairly good, considering the large uncertainties involved in these methods.

As stressed in earlier introductions, developing CG models and simulation schemes for conjugated polymers has been motivated in a large part by the imperative need of resolving supramolecular aggregation properties in solution, and later in quenching thin film. This essential goal, however, poses notable challenges to usual AMD and CGMD simulations for realistic chain lengths. At present, only some preliminary results from CGLD simulation are available, leaving the major discussion and analysis to later subsections, where more efficient simulation schemes are employed to investigate larger-scale aggregation properties. Figures 13 show the snapshots of ten MEH-PPV chains collapsing into a single aggregate cluster in two different solvents. The results provide basic insights into how fast individual chains may coalesce into an aggregate cluster, as well as how the eventual morphologies are dependent on the solvent used. To further characterize the structural compactness of an aggregate cluster, which may be assessed by combined dynamic and static light scatterings, we use the following definition of monomer density:

$$
\rho_{\mathrm{N}}=\frac{N_{\mathrm{b}}}{\frac{4}{3} \pi R_{\mathrm{g}}{ }^{3}},
$$

where $N_{\mathrm{b}}$ and $R_{\mathrm{g}}$ denote the number of monomers and radius of gyration of the cluster, respectively. The monomer density (number of monomers $/ \mathrm{nm}^{3}$ ) is thus estimated to be $\rho_{\mathrm{N}}=0.4$ in chloroform and $\rho_{\mathrm{N}}=0.7$ in toluene. If similar structural features apply for the bulk majority of aggregate species in dilute MEH-PPV solution, the monomer densities so obtained may also be utilized to estimate the mean aggregation number in experiment, which would be difficult, if not impossible, to evaluate from usual light scattering analysis as the commonly practiced Zimm plots cease to be reliable for a highly aggregated system. More details about the simulation protocols, data analysis and discussion can be found elsewhere (Lee et al. 2008)

\subsection{Aggregation properties predicted by ellipsoid-chain models}

The CGLD scheme discussed above, though much more efficient than usual AMD and CGMD simulations, still suffers serious constraints in investigating real large-scale aggregation properties of practical interest. For instance, an ongoing light-scattering study suggests the prevalence of aggregate clusters each consisting of a few tens or even hundreds of chains in dilute MEH-PPV solution. Therefore, more efficient CG models and simulation schemes must be developed in order to facilitate experimental analyses or interpretations. To fulfill this requirement, the CG model considered in Figure 5 or 7 may be exploited. The merits and drawbacks of each of the two CG models have been highlighted in the preceding section, and it is tantalizing to make some comparisons of the predicted aggregation properties. Since a major part of the CGMC simulation based on the ellipsoid-chain model belongs to an ongoing work, only preliminary results are presented in Figure 14, which shows representative single-chain conformations and aggregate morphologies of MEH-PPV in toluene (top left and right) or chloroform (bottom left and right). The monomer densities 
for the aggregates were found to be 0.62 and 0.17 , respectively. Comparing the results with those from the CGLD simulation for 10-chain MEH-PPV systems, there seems to be reasonable agreement between the two, while the monomer densities systematically fall below the values predicted by the CGLD simulation. It could be that the structural compactness decreases with increasing number of polymer chains forming an aggregate cluster, until a critical aggregation number has been reached. Of course, this conjecture awaits further evaluation as simulation results for even larger aggregate clusters become available.

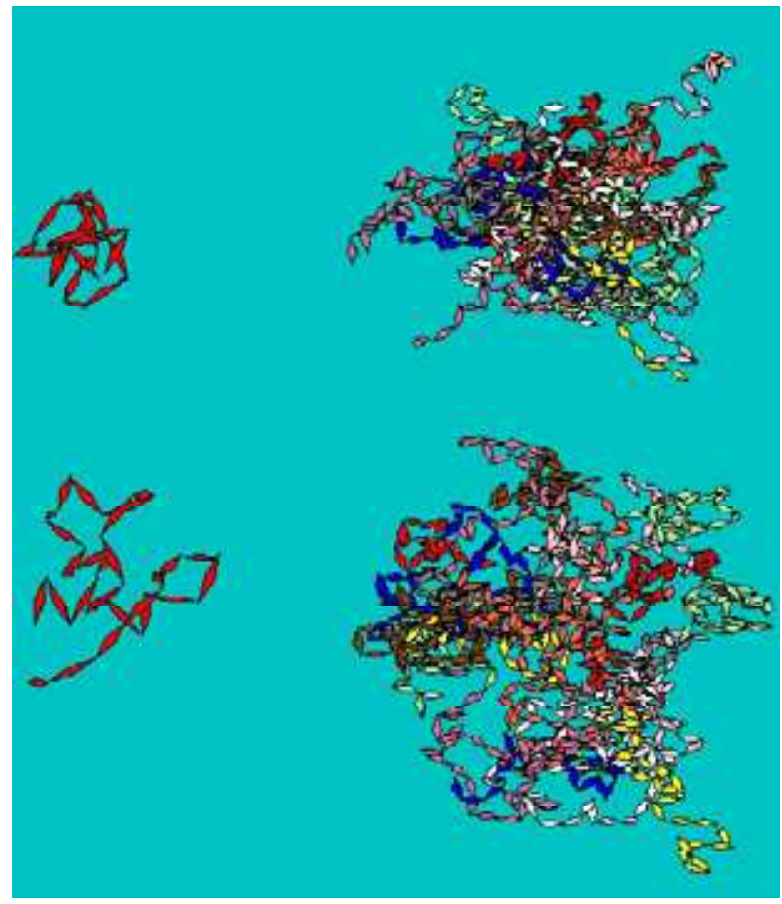

Fig. 14. Realizations of a single, 300-mer MEH-PPV chain (consisting of 30 ellipsoids) based on ellipsoid-chain model in toluene (top-left) or chloroform (bottom-left); realizations of an aggregate cluster made of $20 \mathrm{MEH}-\mathrm{PPV}$ chains in toluene (top-right) or chloroform (bottomright).

\subsection{Comparison with SANS data on single-chain and interchain aggregate structures}

In the literature, the FR chain model and the associated Brownian dynamics schemes have a long history of being employed in studying semiflexible biopolymers such as DNA. To our knowledge, the model has not been employed earlier to study conjugated polymers. Despite a similar level of coarse-graining as with the ellipsoid-chain model considered above, it is possible to carry out usual Brownian dynamics simulations with the FR chain model. The main interest here is twofold: the first is to compare the predicted interchain aggregation properties with what we have briefly reviewed based on the MC simulation of the ellipsoidchain model, and the second is to make direct comparisons with a set of recently reported 
SANS data on MEH-PPV solutions. To simultaneously capture the local rodlike feature and the global coil-like conformation of MEH-PPV, as probed by SANS experiments (Ou-Yang et al. 2005), we have assigned five FR segments to representing a Kuhn segment of MEH-PPV, which is equivalent to treating 10 repeating units of MEH-PPV as one FR segment (Shie et al. 2010) - a CG level identical with the ellipsoid-chain model discussed above. For the sake of simplicity, however, synthesized defects are not considered in the simulation making use of FR chains.
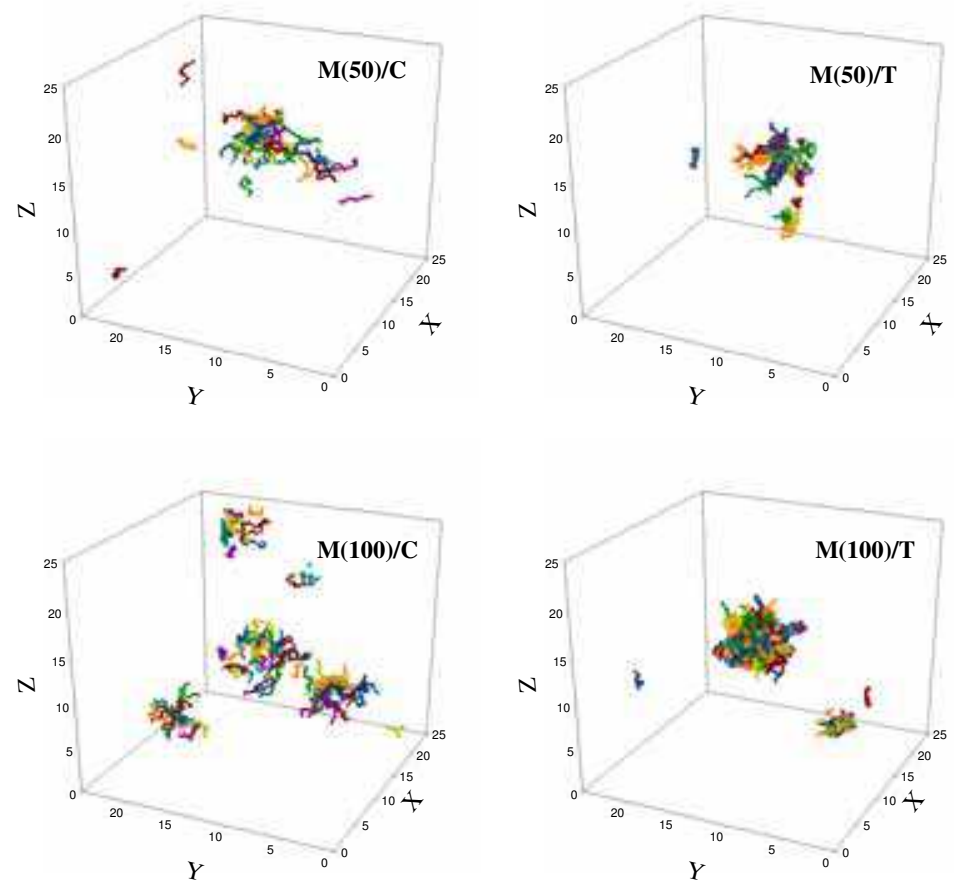

Fig. 15. Snapshots of the aggregation morphologies of MEH-PPV (M) in chloroform (C) or toluene (T) for 50-chain (top) or 100-chain (bottom) system.

Figure 15 shows the results for two many-chain systems consisting of 50 and $100 \mathrm{MEH}-\mathrm{PPV}$ chains, respectively. The simulation starts with randomly distributed isolated chains in a periodic cubic box, and equilibration was judged by eyes to maintain stable aggregation morphologies in both solvent systems. To expedite the simulation, the FJ chains were used to commence the process of aggregation and foster stable aggregate clusters, before a systematic back-mapping was performed to return the system to their children FR chains. Despite the difference in the total number of chains used, remarkable similarity can be noted in the overall aggregation features. While mainly a few compact aggregate clusters are seen for MEH-PPV/toluene system, a larger number of loose aggregate clusters and sporadic isolated chains survive in MEH-PPV/chloroform system. These features, as a whole, are in agreement with the predictions based on the finer-grained models discussed earlier. Thus, the simulation results should allow for a meaningful comparison with the SANS data and, accordingly, render insights not readily perceivable in the SANS data, as we discuss below. 
As has been noted earlier, the FR chain model requires apriori single-chain properties as an input to determine the bonded and non-bonded potentials in general, along with preliminary experimental data to help fix the effective bead diameter in particular. The bead diameter so determined, presumably, should stay insensitive to the solvent considered, and hence, there will remain only one floating parameter readily determinable from a finergrained simulation if a different solvent is to be considered. From this perspective, the model may be regarded as possessing predictive capability for all solvent systems of the same polymer. Figure 16 shows direct theory/data comparisons for a wide range of scattering vectors, $q$. Firstly, one sees a good agreement at large and intermediate values of $q$, where contribution to the total scattering intensity is dominated by the solvent-induced single-chain conformations. The results suggest that interchain aggregates have insignificant influence on single-chain conformations, inasmuch as the structural compactness does not change appreciably after aggregate formation. On the other hand, there exist notable discrepancies in the low $q$ range, where the contribution from interchain aggregates becomes increasingly important.
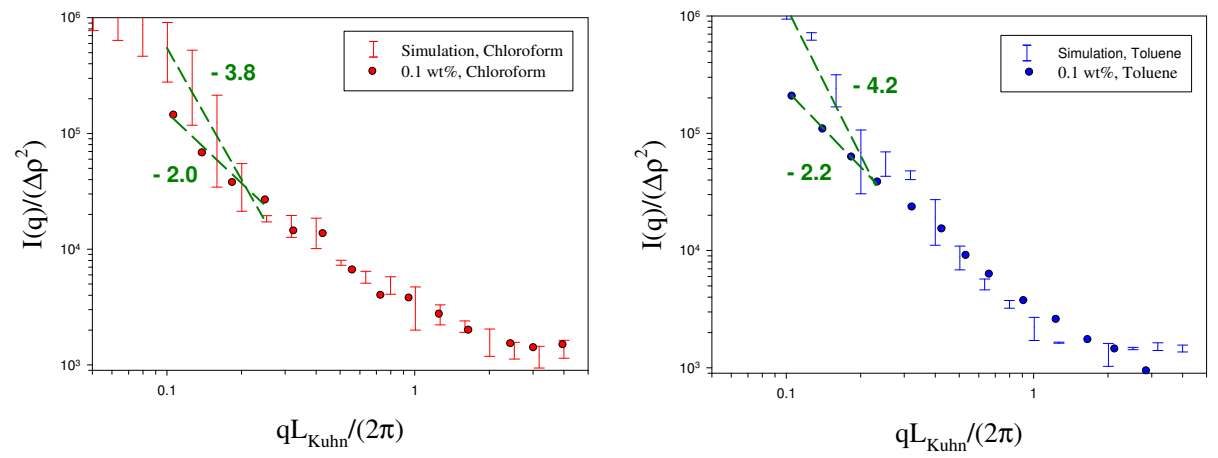

Fig. 16. Comparison between the simulation (using two model aggregate clusters for each solvent system as chosen from the realizations shown in Fig. 15) and SANS data for the total scattering intensity as functions of the scattering vector $q$.

In early experimental interpretations (Ou-Yang et al. 2005), the apparent scaling exponents, ca. 2, in this low- $q$ regime have been assigned to $2 \mathrm{D}$ fractal structures of the aggregation network. The simulation, on the other hand, implies that the nominal scaling exponents could be a consequence of cooperative contributions from a broad variety of aggregate clusters, whose structure remains spherical colloid-like (with a scaling exponent ca. 4), and single, isolated chains, whose contribution was noted to level off but not vanish in this regime. Thus, until even smaller $q$ may be probed, say, by light scattering, the effects of isolated chains and small aggregate clusters - both bear 2D fractal structures-cannot be fully ignored, and the significance of the apparent scaling exponents remains elusive in these cases. Overall, this is an interesting example demonstrating how computer simulations might help resolve issues that otherwise remain ambiguous at an experimental level. To evaluate an earlier proposal concerning structural compactness of the aggregate clusters, we have also examined the monomer density of the largest aggregate cluster in each solvent system, and the results (0.02 for MEH-PPV/chloroform and 0.05 for MEH-PPV/toluene) seem to confirm the expected trends with increasing aggregation number. It should be noted, however, that self-consistency between different CG models awaits to be established before definitive conclusions may be drawn. 


\subsection{Aggregation morphologies predicted by the dumbbell model}

As the main interest in a polymer solution is concerned with the average chain alignment, but not the detailed chain conformation, the dumbbell model depicted in Figure 8-which grasps only polymer end-to-end orientation and represents the most coarse-grained polymer model of all-suffices for this purpose. Figures 17-18 depict the snapshots showing the aggregate formation for 100 such dumbbells that mimic MEH-PPV chains in two different solvents, where the dumbbells at first are randomly placed in the simulation box. The simulation time has been made dimensionless by the estimated Rouse time of the parent FJ chains.

(a) $\mathrm{t} / \tau_{\mathrm{R}}=0$
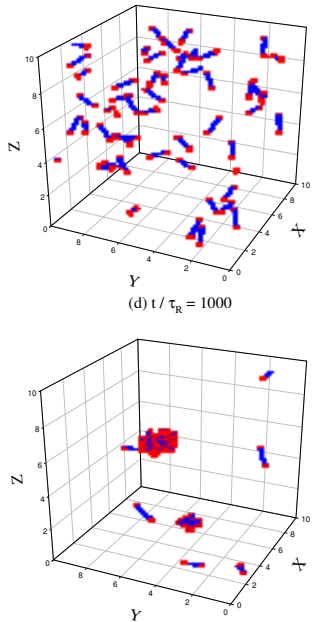

(b) $\mathrm{t} / \tau_{\mathrm{R}}=100$
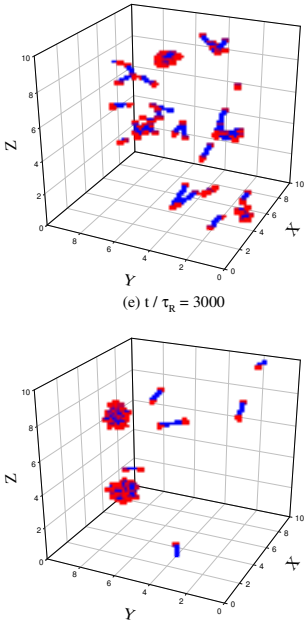

(c) $\mathrm{t} / \tau_{\mathrm{R}}=500$
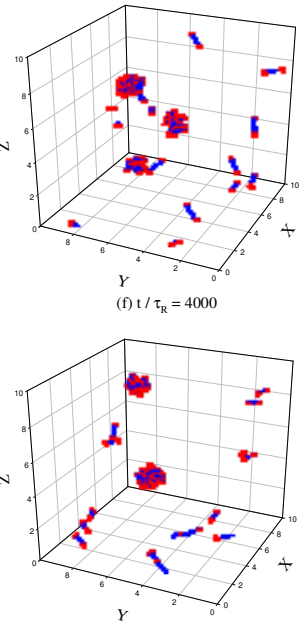

Fig. 17. Snapshots of dynamic aggregation morphologies in MEH-PPV/chloroform solution.

(a) $\mathrm{t} / \tau_{\mathrm{R}}=0$
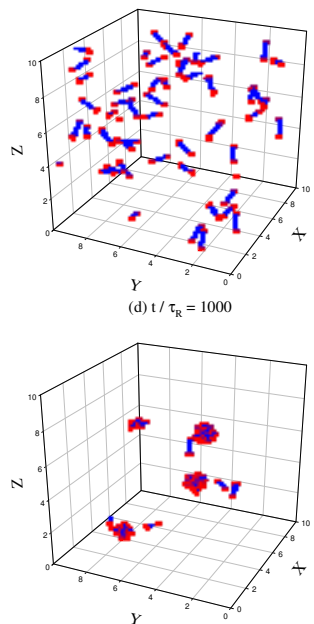

(b) $\mathrm{t} / \tau_{\mathrm{R}}=100$

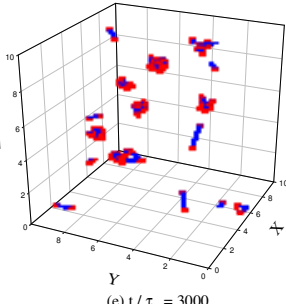

(e) $\mathrm{t} / \tau_{\mathrm{R}}=3000$

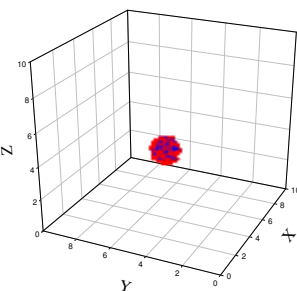

(c) $\mathrm{t} / \tau_{\mathrm{R}}=500$
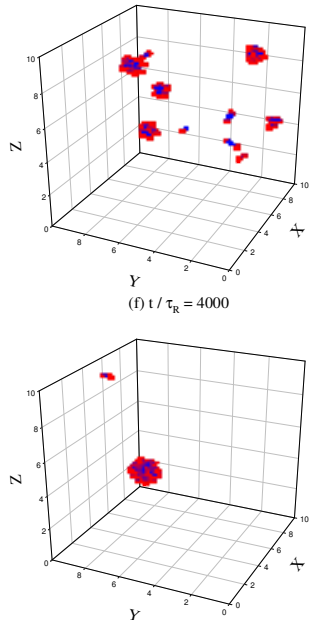

Fig. 18. Snapshots of dynamic aggregation morphologies in MEH-PPV/toluene solution. 
Despite the crudeness of the model used, it is significant to notice a striking similarity with the results shown in Figure 15 based on the more sophisticated FR chain model, in view of the effects of solvent quality on the overall aggregation morphologies. The close agreement seems to suggest the dominance of interchain attractions over the effects of polymer entanglement. More results and discussion can be found elsewhere (Shie et al. 2007), including the influences of a steady shearing that grossly mimics flow processing.

\subsection{Quenched MEH-PPV chain morphologies investigated by reverse mapping}

To shed light on the effects of solvent on the quenched-chain morphologies, the simulation results introduced in Sec. 3.1 for single- and hybrid-solvent systems are systematically backmapped to full-atom coordinates. For simplicity, and to gain general insights, we assume an instant evacuation of solvent molecules, although real film casting involves a more gradual solvent evaporation. The back-mapped, atomistic chains were first allowed to equilibrate for their local structures in the AMD simulation, before the quenching process formally commences. The simulation of chain quenching was carried out in a vacuum, NVT environment at the same system temperature (i.e., $298 \mathrm{~K}$ ), and the time required for a complete chain collapsing has been estimated to be about $2.0 \mathrm{~ns}$ based on the re-scaled, real AMD time. In fact, such an extremely short period compared with the time scales associated with typical film casting might, at least in part, be utilized to justify omitting the effects of solvent evaporation rate.

Figure 19(a)-(b) shows the snapshots of single MEH-PPV chains being quenched from two different solvent media, which have been selected for their stark contrast in the corresponding solvent qualities. It is evident that a previously extended and loose chain conformation, such as that formed in the $\mathrm{CF} / \mathrm{CB}=2: 1$ medium, permits a relatively regular chain folding along the pivotal tetrahedral defects and, in turn, results in a notably higher degree of ordered $\pi-\pi$ stacking, which promotes the electronic delocalization quintessential for local charge transports. Note, in particular, that the eventual quenching structure shown in Figure 19(a) has been arrested by localized, anisotropic $\pi-\pi$ interactions. In contrast, for a previously compact chain conformation, like that formed in toluene and shown in Figure 19(b), the quenched chain ubiquitously becomes much collapsed and featureless, due to the predominant, isotropic van der Waals segmental interactions. Quantitatively, Figure 19(c) (which has been created using time plus ensemble averaging) shows the RDFs revealing the fingerprint of ordered $\pi-\pi$ stacking, which bears a characteristic center-to-center distance about $4.1 \AA$ - or about $3.5 \AA$ for the vertical distance between the two phenyl planes, which is more often referred to-for MEH-PPV chains quenched from various solvent media. Clearly, the chain quenched from the $C F / C B=2: 1$ solvent medium accommodates the greatest amount of $\pi-\pi$ stacking, about 60 percent higher than the second place for $\mathrm{CF} / \mathrm{T}=1: 1$ medium and substantially surpassing the rest single-solvent systems. Moreover, a close correspondence between the mean coil-size (or effective solvent quality) in solution, as previously revealed in Figure 9, and the degree of $\pi-\pi$ stacking in the quenching state can be observed. An interesting exception should be noted, however, with the case of single-solvent CB medium, which apparently results in a higher degree of $\pi-\pi$ stacking despite a smaller mean coil size than in the single-solvent $\mathrm{CF}$ medium. The disparity may be explicated by noting that the CB-induced backbone exposure-as contrasted with the CF-induced side-chain exposure-of MEH-PPV evidently serves as an advantageous precursor for the subsequent formation of $\pi-\pi$ stacking. Thus, not only does the overall chain expansion in solution matter, the detailed solvent-induced chain conformation has an important impact on quenched-chain morphologies as well. In 
future perspective, the quenched chain conformations so obtained may serve as the reference state, based on which quantum chemistry computations may be carried out to further explore the optoelectronic properties of the polymer as quenched from various solvent media. In this way, it should be possible to establish an unequivocal link between the material properties in solution and in quenching thin film.

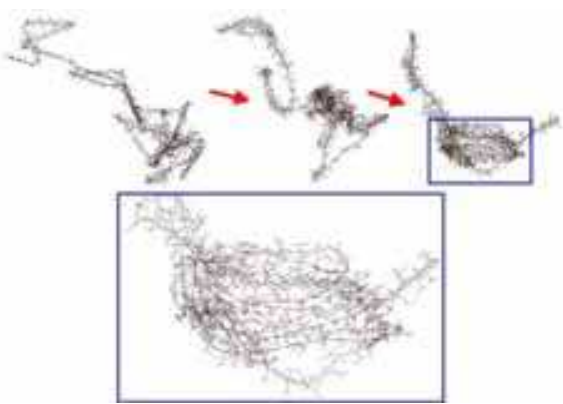

(a)

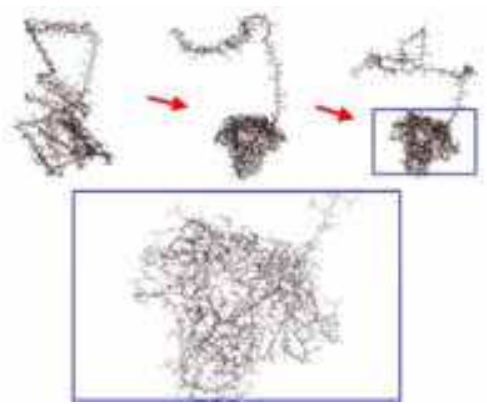

(b)

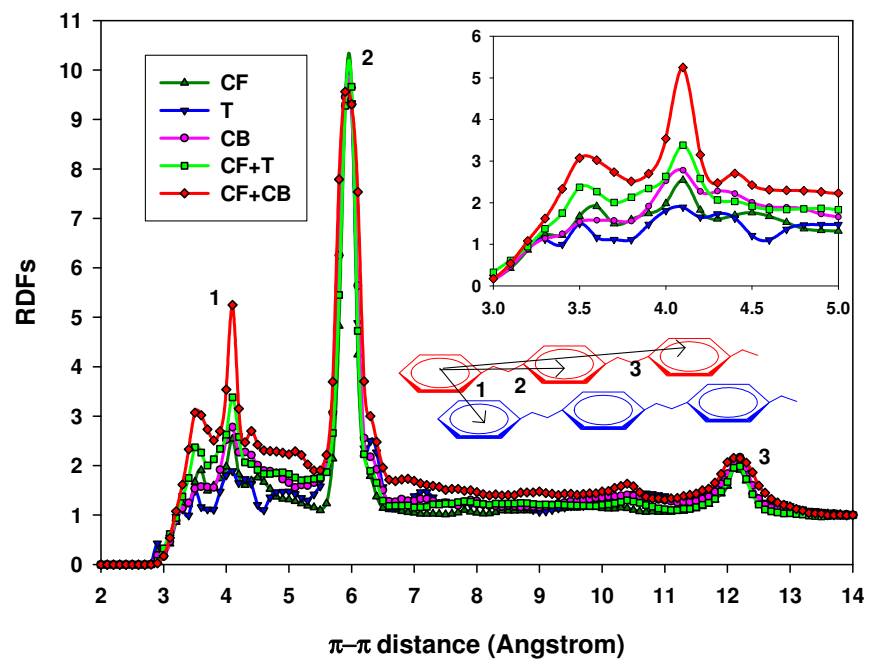

(c)

Fig. 19. Snapshots of a single 300-mer MEH-PPV chain quenched from (a) a binary-solvent medium, $\mathrm{CF} / \mathrm{CB}=2: 1$, and (b) a single-solvent system, $\mathrm{T}$; (c) the RDFs revealing the formation of ordered $\pi-\pi$ stacking for a single MEH-PPV chain quenched from various solvent media, where the inset shows the zoomed plot that best illuminates the relative partition of $\pi-\pi$ stacking (at ca. $4.1 \AA$ ).

\subsection{Phase-separated nanomorphologies in hybrid C60/MEH-PPV system}

An emerging area that shall find profound applications of CG simulations is to unravel the detailed mechanisms regulating nanoscale phase-separated morphologies of hybrid fullerene/conjugated polymer systems, which have received growing attention recently in 
fabricating heterojunction thin films for application in organic solar cells. Among them, C60/MEH-PPV has since served as a primitive model system to unveil how the aforementioned nanomorphologies impact the optoelectronic properties of a solar cell device. At a preliminary stage, we have obtained results as shown in Figure 20 using the interparticle potential found in an early CGMD simulation of C60 (Izvekov et al. 2005) and recast into a $\mathrm{LJ}$ form. For the $\mathrm{TiO}_{2}$ nanorods, for example, deployed as an electrode for conducting the electrons to the outer circuit, the potential functions established in a recent multiscale computation on silica nanoparticles (Lee \& Hua 2010) were directly transplanted onto the building blocks of $\mathrm{TiO}_{2}$ to model the interactions with $\mathrm{C} 60$ or MEH-PPV. An intriguing feature in Figure 20 is the appearance of phase-separated, yet interconnected, nano-domains that are crucial for the transports of light-triggered electrons or holes. Ongoing studies utilizing more accurate potential functions will systematically explore the impacts of several essential factors, including the blending ratio and the solvent medium, on the eventual nanomorphologies of similar fullerene/conjugated polymer mixtures.
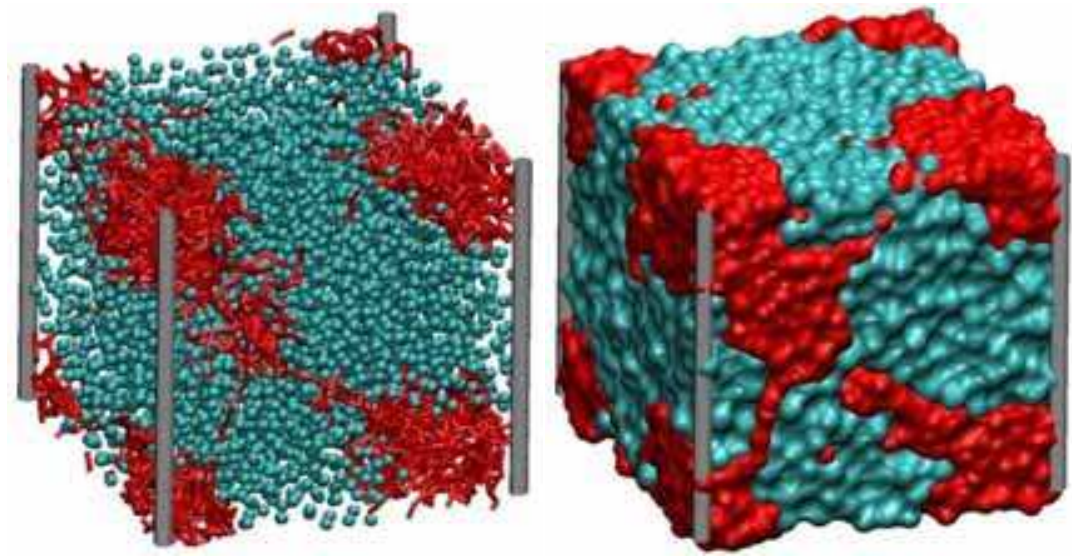

Fig. 20. Phase-separated nanomorphologies, sketched in two different perspectives, of a hybrid C60(dots)/MEH-PPV(fuzzy lines in the left picture) system with the presence of four vertical nanorods made of $\mathrm{TiO}_{2}$.

\section{Conclusion}

This chapter reviews recent progresses in developing self-consistent, parameter-free CG models and simulation schemes aimed to capture fundamental single-chain and aggregation properties of standard conducting conjugated polymers from solution to the quenching state. Molecular insights so gained are essential to complement our current knowledge from conventional experimental characterizations, which become ineffective, if not impossible, in resolving nanoscale material properties of single chains and aggregate clusters in various solvent media and quenching processes. We demonstrated through these simulations how an unequivocal link between the material properties in solution and in the quenching state may be established, and remarked on the promising prospects of making direct predictions on the optoelectronic behavior of a particular polymer quenched from specific solvents or hybrid solvents. Progress along this line should help guide future molecular designs and process controls that best meet the quest of maneuvering the device performances at nanoscale levels. It should be reemphasized, however, that the strategies proposed and the applications 
discussed should not be restricted to the particular polymer or solvent species considered, and the hierarchy of CG models and simulation schemes introduced in this monographpresumably, starting from quantum-level computations to the construction of predictive dumbbell models - may be dexterously exploited in conjunction with the forward/backward mapping schemes to resolve material properties of practical interest. In future perspectives, these multiscale schemes may be automated in such a way that, given a specific polymersolvent pair, interested material properties can be assessed in advance, subject only to the limits of contemporary computing resources. The prime challenges, however, seem to so far lie in the difficulties of treating anisotropic, highly localized intermolecular potentials, such as hydrogen-bond forces and $\pi-\pi$ interactions, in any CG models beyond the atomistic level. This situation would imply that such interaction forces, quintessential in dense or condensed states, may be realistically accounted for at the current stage only through a series of back mappings to the atomistic level. A recent study, nonetheless, suggested that these localized interaction forces might play only minor roles in solution state (Lee et al. 2009), for which the demand of CG simulations is among the most stringent. Another appealing outlook for exploiting multiscale computation schemes is the emerging possibility to treat the interactions between fullerene/polymer and a solid substrate (Lee \& Hua 2010), as hinted by the last example discussed with Fig. 20. Fulfilling this objective should make the current applications on thin-film devices more complete and powerful. Of course, it remains an essential task to ensure self-consistencies between various CG models and simulation schemes, and there still remains plenty room for future refinements. In conclusion, the seemingly concurrent advances in contemporary multiscale computations and organic optoelectronics have brought in not only strong motivations but also new opportunities for researchers who find themselves enthralled by, and to practically benefit from, an increasing interplay of the two.

\section{Acknowledgment}

The authors thank the supports from the National Science Council of ROC as well as the resources provided by the National Center for High-Performance Computing.

\section{References}

Carbone, P.; Karimi-Varzaneh, H. A., \& Müller-Plathe, F. (2010). Fine-Graining without Coarse-Graining: An Easy and Fast Way to Equilibrate Dense Polymer Melts. Faraday Discussions, Vol. 144, No. pp. 25-42, ISSN 1364-5498.

De Leener, C.; Hennebicq, E.; Sancho-Garcia, J. C., \& Beljonne, D. (2009). Modeling the Dynamics of Chromophores in Conjugated Polymers: The Case of Poly (2methoxy-5-(2'-ethylhexyloxy)-1,4-phenylenevinylene) (MEH-PPV). eburnal of Physical Chemistry B, Vol. 113, No. 5, pp. 1311-1322, ISSN 1520-6106.

Faller, R. (2004). Automatic Coarse Graining of Polymers. Polymer, Vol. 45, No. 11, pp. 38693876, ISSN 0032-3861.

Forester, T. R., \& Smith, W. (2006). The DL_POLY_2 Reference Manual, Laboratory Daresbury, Daresbury.

Gay, J. G., \& Berne, B. J. (1981). Modification of the Overlap Potential to Mimic a Linear SiteSite Potential. eburnal of Chemical Physics, Vol. 74, No. 6, pp. 3316-3319, ISSN 00219606.

Gettinger, C. L.; Heeger, A. J.; Drake, J. M., \& Pine, D. J. (1994). A Photoluminescence Study of Poly(phenylene vinylene) Derivatives: the Effect of Intrinsic Persistence Length. cburnal of Chemical Physics, Vol. 101, No. 2, pp. 1673-1678, ISSN 0021-9606. 
Izvekov, S.; Violi, A., \& Voth, G. A. (2005). Systematic Coarse-Graining of Nanoparticle Interactions in Molecular Dynamics Simulation. eburnal of Physical Chemistry B, Vol. 109, No. 36, pp. 17019-17024, ISSN 1520-6106.

Lee, C. K., \& Hua, C. C. (2010). Nanoparticle Interaction Potentials Constructed by Multiscale Computation. eburnal of Chemical Physics, Vol. 132, No. 22, pp. 224904, ISSN 0021-9606.

Lee, C. K.; Hua, C. C., \& Chen, S. A. (2008). Single-Chain and Aggregation Properties of Semiconducting Polymer Solutions Investigated by Coarse-Grained Langevin Dynamics Simulation. eburnal of Physical Chemistry B, Vol. 112, No. 37, pp. 1147911489, ISSN 1520-6106.

Lee, C. K.; Hua, C. C., \& Chen, S. A. (2009). Multiscale Simulation for Conducting Conjugated Polymers from Solution to the Quenching State. eburnal of Physical Chemistry B, Vol. 113, No. 49, pp. 15937-15948, ISSN 1520-6106.

Lee, C. K.; Hua, C. C., \& Chen, S. A. (2010). Parametrization of the Gay-Berne Potential for Conjugated Oligomer with a High Aspect Ratio. eburnal of Chemical Physics, Vol. 133, No. 6, pp. 064902, ISSN 0021-9606.

Lee, C. K.; Hua, C. C., \& Chen, S. A. (2011). Hybrid Solvents Incubated п-п Stacking in Quenched Conjugated Polymer Resolved by Multiscale Computation. Macromolecules, Vol. 44, No. 2, pp. 320-324, ISSN 0024-9297.

Mayo, S. L.; Olafson, B. D., \& Goddard, W. A. (1990). Dreiding-A Generic Force-Field for Molecular Simulations. eburnal of Chemical Physics, Vol. 94, No. 26, pp. 8897-8909, ISSN 0022-3654.

Müller-Plathe, F. (2002). Coarse-Graining in Polymer Simulation: from the Atomistic to the Mesoscopic Scale and Back. Chemphyschem, Vol. 3, No. 9, pp. 754-769, ISSN 1439-4235.

Nguyen, T. Q.; Doan, V., \& Schwartz, B. J. (1999). Conjugated Polymer Aggregates in Solution: Control of Interchain Interactions. eburnal of Chemical Physics, Vol. 110, No. 8, pp. 4068-4078, ISSN 0021-9606.

Noid, W. G.; Chu, J. W.; Ayton, G. S.; Krishna, V.; Izvekov, S.; Voth, G. A.; Das, A., \& Andersen, H. C. (2008). The Multiscale Coarse-Graining Method. I. A Rigorous Bridge between Atomistic and Coarse-Grained Models. eburnal of Chemical Physics, Vol. 128, No. 24, pp. 244114, ISSN 0021-9606.

Ou-Yang, W. C.; Chang, C. S.; Chen, H. L.; Tsao, C. S.; Peng, K. Y.; Chen, S. A., \& Han, C. C. (2005). Micellelike Aggregates in Solutions of Semirigid Hairy-Rod Polymers. Physical Review E, Vol. 72, No. 3, pp. 031802, ISSN 1550-2376.

Padding, J. T., \& Briels, W. J. (2011). Systematic Coarse-Graining of the Dynamics of Entangled Polymer Melts: the Road from Chemistry to Rheology. In: Soft Condensed Matter, Available from: arxiv.org/pdf/1102.0732.

Shie, S. C.; Hua, C. C., \& Chen, S. A. (2007). Brownian Dynamics Simulation of SelfConsistently Coarse-Grained Dilute Aggregated Polymer Suspensions. Macromolecular Theory and Simulations, Vol. 16, No. 1, pp. 111-122, ISSN 1022-1344

Shie, S. C.; Lee, C. K.; Hua, C. C., \& Chen, S. A. (2010). A Predictive Coarse-Grained Model for Semiflexible Polymers in Specific Solvents. Macromolecular Theory and Simulations, Vol. 19, No. 4, pp. 179-189, ISSN 1022-1344

Tschöp, W.; Kremer, K.; Batoulis, J.; Bürger, T., \& Hahn, O. (1998). Simulation of Polymer Melts. I. Coarse-Graining Procedure for Polycarbonates. Acta Polymerica, Vol. 49, No. 2-3, pp. 61-74, ISSN 1521-4044. 


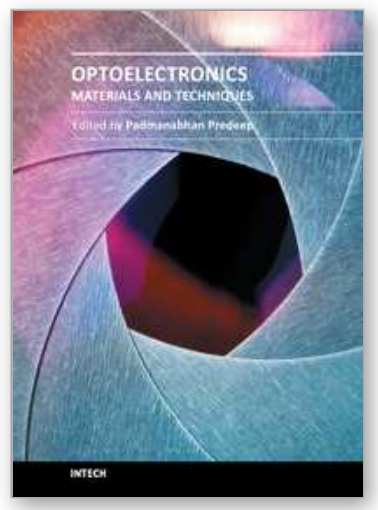

\author{
Optoelectronics - Materials and Techniques \\ Edited by Prof. P. Predeep
}

ISBN 978-953-307-276-0

Hard cover, 484 pages

Publisher InTech

Published online 26, September, 2011

Published in print edition September, 2011

Optoelectronics - Materials and Techniques is the first part of an edited anthology on the multifaceted areas of optoelectronics by a selected group of authors including promising novices to the experts in the field.

Photonics and optoelectronics are making an impact multiple times the semiconductor revolution made on the quality of our life. In telecommunication, entertainment devices, computational techniques, clean energy harvesting, medical instrumentation, materials and device characterization and scores of other areas of R\&D the science of optics and electronics get coupled by fine technology advances to make incredibly large strides. The technology of light has advanced to a stage where disciplines sans boundaries are finding it indispensable. Smart materials and devices are fast emerging and being tested and applications developed in an unimaginable pace and speed. Here has been made an attempt to capture some of the materials and techniques and underlying physical and technical phenomena that make such developments possible through some real time players in the field contributing their work and this is sure to make this collection of essays extremely useful to students and other stake holders such as researchers and materials scientists in the area of optoelectronics.

\title{
How to reference
}

In order to correctly reference this scholarly work, feel free to copy and paste the following:

Cheng K. Lee and Chi C. Hua (2011). Nanomorphologies in Conjugated Polymer Solutions and Films for Application in Optoelectronics, Resolved by Multiscale Computation, Optoelectronics - Materials and Techniques, Prof. P. Predeep (Ed.), ISBN: 978-953-307-276-0, InTech, Available from: http://www.intechopen.com/books/optoelectronics-materials-and-techniques/nanomorphologies-in-conjugatedpolymer-solutions-and-films-for-application-in-optoelectronics-resolv

\section{INTECH}

open science | open minds

\author{
InTech Europe \\ University Campus STeP Ri \\ Slavka Krautzeka 83/A \\ 51000 Rijeka, Croatia \\ Phone: +385 (51) 770447 \\ Fax: +385 (51) 686166 \\ www.intechopen.com
}

\author{
InTech China \\ Unit 405, Office Block, Hotel Equatorial Shanghai \\ No.65, Yan An Road (West), Shanghai, 200040, China \\ 中国上海市延安西路65号上海国际贵都大饭店办公楼 405 单元 \\ Phone: +86-21-62489820 \\ Fax: +86-21-62489821
}


(C) 2011 The Author(s). Licensee IntechOpen. This chapter is distributed under the terms of the Creative Commons Attribution-NonCommercialShareAlike-3.0 License, which permits use, distribution and reproduction for non-commercial purposes, provided the original is properly cited and derivative works building on this content are distributed under the same license. 